\title{
The difference of intraoperative free-run electromyography monitoring between percutaneous endoscopic lumbar discectomy via a transforaminal and via an interlaminal
}

\author{
Jun-Ichiro Nakamura, Tomoyuki Setoue, Jun Hara \\ Orthopedic surgery, Kawasaki-saiwai hospital, Kawasaki city, Kanagawa-ken 212-0014, Japan.
}

Correspondence to: Dr. Jun-Ichiro Nakamura, Orthopedic surgery, Kawasaki-saiwai hospital, 31-27 Ohmiya-cho, Saiwai-ku, Kawasaki city, Kanagawa-ken 212-0014, Japan. E-mail: nakajun16@yahoo.co.jp

\begin{abstract}
How to cite this article: Nakamura JI, Setoue T, Hara J. The difference of intraoperative free-run electromyography monitoring between percutaneous endoscopic lumbar discectomy via a transforaminal and via an interlaminal. Mini-invasive Surg 2019;3:29. http://dx.doi.org/10.20517/2574-1225.2019.28
\end{abstract}

Received: 26 Aug 2019 First Decision: 26 Sep 2019 Revised: 2 Oct 2019 Accepted: 10 Oct 2019 Published: 15 Oct 2019

Science Editor: Giulio Belli Copy Editor: Cai-Hong Wang Production Editor: Tian Zhang

\begin{abstract}
Aim: Transforaminal percutaneous endoscopic lumbar discectomy (TF-PELD) is usually performed under local anesthesia because the patient should be conscious to prevent nerve root injury. However, some patients cannot tolerate intraoperative pain and require intravenous analgesia, or must be converted to surgery under general anesthesia (GA). If PELD under GA can be performed safely, it is more convenient and comfortable for both the patient and surgeon.
\end{abstract}

Methods: A total of 49 cases (mean age, 53 years) were examined. PELD was performed under GA with free-run electromyography (f-EMG) monitoring. Clinical outcomes were assessed according to the visual analogue scale score (VAS) and the Oswestry disability index (ODI). All patients were monitored with f-EMG.

Results: VAS decreased from 7.7 to 1.1 and ODI from $62.3 \%$ to $20.5 \%$. A true-positive was observed in one of 27 TFPELD cases. Care during the procedure is necessary to avoid the risk of severe neurological injury. A false-negative was observed in one of 22 interlaminar (IL)-PELD cases. This patient complained of aggravated numbness for 6 months after surgery. False-positives were recorded in 2 cases of IL-PELD with a train wave just after removal of the herniated discs.

Conclusion: F-EMG monitoring during PELD under GA was useful to identify nerve root damage. TF-PELD under GA requires f-EMG to ensure safety. On the contrary, IL-PELD does not necessitate f-EMG.

Keywords: Free-run electromyography, general anesthesia, iatrogenic nerve injury, percutaneous endoscopic lumbar discectomy

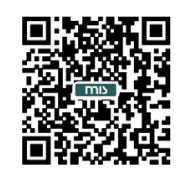




\section{INTRODUCTION}

Transforaminal percutaneous endoscopic lumbar discectomy (TF-PELD) is usually performed on an outpatient basis under local anesthesia (LA). The procedure is neurologically safe and avoids injury to the exiting nerve root (ENR) since the patient is conscious and able to notify the surgeon of any pain ${ }^{[1-6]}$.

However, some patients cannot tolerate intraoperative pain and may acquire intravenous analgesia, sedative agents, or conversion to epidural or general anesthesia (GA) on another day.

PELD under GA is considered neurologically safe since the patient is unconscious. Recently, the efficacy of free-run electromyography (f-EMG) has been reported in neurosurgery and spine surgery ${ }^{[7-12]}$, but not for PELD. Therefore, the aim of this study was to evaluate the efficacy of f-EMG monitoring during PELD under GA.

\section{METHODS}

\section{Anesthesia}

Propofol and remifentanil hydrochloride-based GA were administered. A muscle relaxant (rocuronium bromide) was only used at the initial stage for intubation, and sugammadex sodium was used for reversal of neuromuscular blockade. The stimulation pattern (i.e., train-of-four) was confirmed as more than $90 \%$ before starting of f-EMG monitoring.

\section{F-EMG}

Continuous f-EMG activity was recorded using a NVM5 ${ }^{\circ}$ monitoring system (NuVasive Inc., San Diego, CA, USA) with surface electrode patches placed at 6 positions (i.e., the bilateral vastus mediaris, tibialis anterior, and gastrocnemius). Before affixing the patches, the skin was scraped with sandpaper to ensure impedance was less than $10 \mathrm{k} \Omega$. The threshold of electrical impact was set at $80 \mu \mathrm{V}$. All instances of f-EMG activity were immediately reported to the surgeon.

Neurotonic, burst, and train waves that occurred in response to a surgical maneuver to the affected nerve were considered to be abnormal. A small amplitude, low frequency, and isolated discharge were not considered pathologic $^{[10]}$. Any of these instances automatically sounded an alarm to notify the surgeon.

\section{Surgical procedure}

\section{TF-PELD}

With the patient in the prone position on a radiolucent table, an 8 - $\mathrm{mm}$ incision was made to the entry points of the skin at about 9-11 cm lateral to the midline and then an 18-gauge needle was inserted into the annulus fibrosus. Discography with $2 \mathrm{~mL}$ of liquid indigo carmine contrast agent (1:1) was performed using the same needle. A guide wire and sleeve were inserted for endoscopy with a VERTEBRIS lumbarthoracic ${ }^{\bullet}$ instrument (Richard Wolf $\mathrm{GmbH}$, Knittlingen, Germany). A bipolar radio-frequency electrode system (Elliquence, Baldwin, NY, USA) was used for hemostasis and a high-speed Primado $2^{\circ}$ drill with a super slim hand piece $200^{\circ}$ (NSK-Nakanishi medical, Tochigi, Japan) was used for foraminoplasty.

\section{IL-PELD}

With the patient in the prone position on a radiolucent table, an $8-\mathrm{mm}$ incision was made to the entry points of the skin at about $1 \mathrm{~cm}$ lateral to the midline. Once the entry point and the direction of the sleeve were confirmed by image intensifier, a 8 -mmsleeve was inserted. A VERTEBRIS lumbar-thoracic ${ }^{\circ}$ instrument was used for endoscopy and a bipolar radio-frequency electrode system was also used for hemostasis. The ligamentum flavum was resected piece-by-piece using a micro punch. A high-speed Primado $2^{\circ}$ drill was used for laminotomy and medial facetectomy. Partial laminotomy was necessary in almost all cases, particularly at L4-L5, to remove the laminae and ligamentum flavum and confirm the 


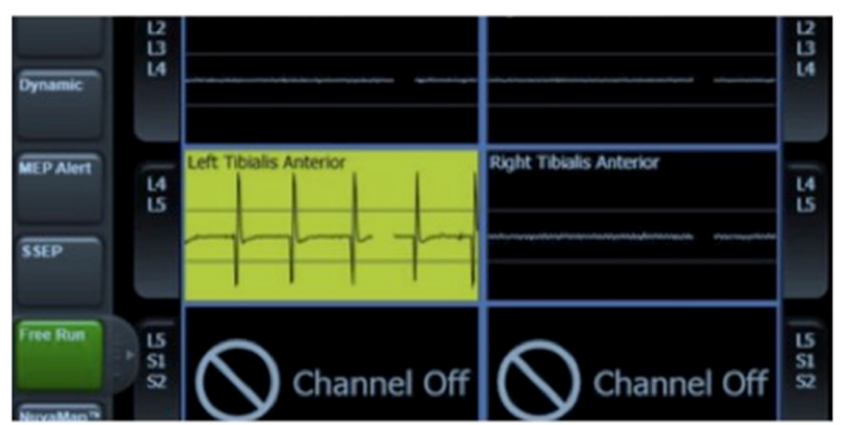

Figure 1. Train wave of free-run electromyogram. This wave was observed in TF-PELD at L4-L5 case when the sleeve was manipulated hand-down. Same waves were also observed in IL-PELD at L5-S1. TF-PELD: transforaminal percutaneous endoscopic lumbar discectomy; IL: interlaminar

lateral edge of the nerve root. Then, after the sleeve was rotated and thecal sac was retracted, the herniated disc was removed.

\section{Statistical analysis}

Descriptive analysis of group characteristics was performed using JMP version 11.2 software for Macintosh (SAS Institute Inc., Cary, NC, USA). The independent two-sample $t$-test and Wilcoxon test were used to compare the clinical outcomes. A probability value of $P<0.05$ was considered to be statistically significant.

\section{RESULTS}

There were 49 patients ( 43 men, 6 women) with a mean age of 52.9 (range, 17-88) years. The mean followup period was 10 (range, 6-14) months. The affected level was L2-L3 in one patient (TF-PELD), L3-L4 in 9 (TF-PELD), L4-L5 in 17 [TF-PELD, 14; interlaminar (IL)-PELD, 3], and L5-S1 in 22 (TF-PELD, 3; ILPELD, 19) patients.

The mean operative time was $63 \pm 29$ (range, 30-143) min and intraoperative blood loss was negligible in all cases. The mean hospital stay was $3.2 \pm 1.5$ (range, 1-5) days. The mean numerical rating scale score for the affected leg improved significantly from 7.7 to 1.1 at follow-up, and the mean Oswestry disability index had improved from 62.3 to 20.5. Two patients experienced recurrence of the herniated nucleus pulposus.

In all cases, single waves were observed but were not considered to be clinically significant. A truepositive was observed in one case during TF-PELD at L4-L5 [Figure 1]. When the sleeve was manipulated downward by hand, an alarm sounded. Careful performance of the procedure will prevent ringing of the alarm, which indicates a postoperative motor deficit. However, this patient complained of dysesthesia for 3 weeks postoperatively. The numbness gradually improved with the use of pregabalin and disappeared by the final follow-up.

False-positives were observed in 2 patients following IL-PELD at L5-S1. No alarm sound was observed when the nerve root was retracted by rotating the sleeve. Train waves appeared with the alarm several seconds after the herniated disc material was removed. At that time, no surgical maneuver was performed. In these 2 cases, no neurological deficit was observed after surgery. Including these 2 cases, no alarm sounded during IL-PELD when the nerve root and dura were retracted.

A false-negative was observed in one patient following IL-PELD at L5-S1, but no abnormal wave was observed. However, this patient complained of severe numbness for 6 months postoperatively. 
The sensitivity of f-EMG for neurological deficits was $50 \%$ in all cases, $100 \%$ for TF-PELD, and $0 \%$ for ILPELD. The specificity was $95.8 \%, 100 \%$, and $90.4 \%$, respectively.

\section{DISCUSSION}

F-EMG's effectiveness has been reported for cranial nerve tumor resection and spinal surgery ${ }^{[7-11]}$. Any irritation to the nerve, by stretching or compression, causes trains of motor unit potential discharge in the corresponding muscle ${ }^{[7]}$. F-EMG is reported to have high sensitivity and relatively low specificity ${ }^{[8]}$. However, monitoring in real-time would improve specificity when confirming that the related nerve was correctly manipulated during surgery ${ }^{[7]}$. Therefore, application of f-EMG in PELD surgery is considered to be efficient.

One of the most important complications in TF-PELD is ENR injury, which reportedly occurs at a relatively high rate of $2 \%-8.9 \%$ under $\mathrm{LA}^{[3,4,13-16]}$. A large LA dosage may increase the risk of ENR injury because of complete nerve blockage. Furthermore, some patients cannot tolerate surgery because of pain and, thus, had to be converted to GA on another day. Therefore, we believe that PELD under LA is not necessarily safe or comfortable for the patient.

In this study, there was one true-positive case with detectable nerve irritation during surgery. This patient complained of severe numbness without motor deficit after surgery. Train waves appeared when the sleeve was manipulated by hand. Hence, caution is required during surgery to avoid motor deficits.

Conversely, we carefully monitored f-EMG during IL-PELD while rotating the sleeve when retracting the dural sac and nerve root. No alarm sounded when performing IL-PELD. However, 2 false-positives were observed during IL-PELD. An alarm sounded after herniated disc material was removed. F-EMG sensitivity during IL-PELD was low. Hence, f-EMG may be inappropriate for monitoring the nerve root during ILPELD.

F-EMG monitoring cannot detect damage to sensory nerves, but it can potentially prevent injury to motor and sensory fibers. PELD under GA, without the use of a muscle relaxant with f-EMG monitoring, can reduce neural injury.

This study was limited by the small number of patients. Therefore, future studies with larger numbers of patients will be necessary to evaluate the efficacy of f-EMG for PELD.

In conclusion, PELD was performed safely under GA using f-EMG monitoring. Patients who cannot tolerate pain are good candidates for PELD under GA.

\section{DECLARATIONS}

\section{Acknowledgments}

The authors would like to thank Enago (www.enago.jp) for the English language review.

\section{Authors' contributions}

Wrote and reviewed the manuscript: Nakamura JI, Setoue T, Hara J

\section{Availability of data and materials}

Not applicable.

\section{Financial support and sponsorship}

None. 


\section{Conflicts of interest}

All authors declared that there are no conflicts of interest.

\section{Ethical approval and consent to participate}

Not applicable.

\section{Consent for publication}

Not applicable.

\section{Copyright}

(c) The Author(s) 2019.

\section{REFERENCES}

1. Kambin P, Schaffer JL. Percutaneous lumbar discectomy. Review of 100 patients and current practice. Clin Orthop Rel Res 1989;238:24-34.

2. Yeung AT, Tsou PM. Posterolateral endoscopic excision for lumbar disc herniation. Surgical technique, outcome, and complications in 307 consecutive cases. Spine 2002;27:722-31.

3. Yeung AT, Yeung CA. Minimally invasive techniques for the management of lumbar disc herniation. Orthop Cl North Am 2007;38:36372.

4. Choi G, Lee SH, Raiturker PP, Lee SS, Chae YS. Percutaneous endoscopic interlaminar discectomy for intracanalicular disc herniations at L5-S1 using a rigid working channel endoscope. Neurosurg 2006;58:59-68.

5. Ruetten S, Komp M, MerkH, Godolias G. Full-endoscopic interlaminar and transforaminal lumbar discectomy versus conventional microsurgical technique: a prospective randomized, control study. Spine 2008;33:931-9.

6. Sairyo K, Egawa H, Matsuura T, Takahashi M, Higashino K, et al. State of the art: transforaminal approach for percutaneous endoscopic lumbar discectomy under local anesthesia. J Med Invest 2014;61:217-25.

7. Pajewski TN, Arlet V, Phillips LH. Current approach on spinal cord monitoring: the point of view of the neurologist, the anesthesiologist and the spine surgeon. Eur Spine J 2007;16:S115-29.

8. Holmes JT, Chappuis JL. Monitoring of lumbosacral nerve roots during spinal instrumentation. Spine 1993;18:2059-62.

9. Skinner SA, Nagib M, Bergman TA, Maxwell RE, Msangi G. The initial use of free-running electromyography to detect early motor tract injury during resection of intramedullary spinal cord lesions. Neurosurg 2005;56:299-314; discussion 299-314.

10. Gunnarsson T, Krassioukov AV, Sarjeant R, Fehlings MG. Real-time continuous intraoperative electromyographic and somatosensory evoked potential recordings in spinal surgery: correlation of clinical and electrophysiologic findings in a prospective, consecutive series of 213 cases. Spine 2004;29:677-84.

11. Chung I, Grigorian A. EMG and evoked potentials in the operating room during spinal surgery. In: Schwartz M, editor. EMG methods for evaluating muscle and nerve function. InTech; 2011. pp. 325-40.

12. Nakamura JI, Yoshihara K. Initial clinical outcomes of percutaneous full-endoscopic lumbar discectomy using an interlaminar approach at the L4-L5. Pain Physician 2017;20:E507-12.

13. Sairyo K, Matsuura T, Higashino K, Sakai T, Takata Y, et al. Surgery related complications in percutaneous endoscopic lumbar discectomy under local anesthesia. J Med Invest 2014;61:264-9.

14. Sakane M. Anatomical relationship between Kambin's triangle and exiting nerve root. Mini-invasive Surg 2017;1:99-102.

15. Fang G, Ding Z, Song Z. Comparison of the effects of epidural anesthesia and local anesthesia in lumbar transforaminal endoscopic surgery. Pain Physician 2016;19:E1001-4.

16. Choi I, Ahn JO, So WS, Lee SJ, Choi IJ, et al. Exiting root injury in transforaminal endoscopic discectomy: preoperative image considerations for safety. Eur Spine J 2013;22:2481-7. 